\title{
The validity of Es/Ep as a source parameter in mining seismology
}

\author{
I.G. Morkel, J. Wesseloo, and Y. Potvin
}

Australian Centre for Geomechanics, Australia

\begin{abstract}
It is generally accepted that the ratio of energy associated with the S-wave (Es) and P-wave (Ep) is dependent on the focal mechanism (Mendecki 2013). In the mining industry, the ratio of S-wave energy to P-wave energy is regarded as an important indicator of the type of focal mechanism, with the ratio being lower for explosive sources and higher for fault slip (Cai et al 1998, Mendecki, 2013). In pure shear, the Es is considerably larger than Ep (Es/Ep > 20). For the tensile model, Sato (1978) has shown that Ep and Es are approximately equal. Gibowicz et al (1991) and Gibowicz and Kijko (1994) suggest that when Es/Ep < 10, the source mechanism involves a tensile failure component. Boatwright and Fletcher (1984) suggest pure shear to correspond with Es/Ep > 10. Hudyma and Potvin (2010) suggest that for events with Es/Ep <3, the mechanism is non-shear.
\end{abstract}

This paper investigates the Es/Ep ratio parameter and how sensitive it is to different seismic service setups. It will achieve this by investigating the consistency of the parameter for three different scenarios.

\section{INTRODUCTION}

It is generally accepted that the ratio of energy associated with the Es/Ep is dependent on the focal mechanism. It is regarded as an important indicator of the type of focal mechanism, with the ratio being lower for explosive sources and higher for fault slip (Mendecki (2013). In the mining industry, this parameter is often used for diagnostic purposes of which some recent examples include Sweby et al (2006), McGaughey et al (2007), Wesseloo and Sweby (2008), Reyes-Montes et al (2010), Disley (2014), McGaughey (2014), Rebuli and Kohler (2014), Abolfazlzadeh et al (2017), Nordström et al (2017), Abolfazlzadeh et al (2019).

In pure shear, the Es is considerably larger than Ep (Es/Ep > 20). For the tensile model, Sato (1978) has shown that Ep and Es are approximately equal. Gibowicz et al (1991) and Gibowicz and Kijko (1994) suggest that when Es/Ep <10, the source mechanism involves a tensile failure component, and Boatwright and Fletcher (1984) suggest pure shear corresponds with Es/Ep > 10. Hudyma and Potvin (2010) suggest that for events with Es/Ep $<3$ the mechanism is non-shear.

This paper reports on a systematic study performed to investigate and quantify the consistency and transferability of the Es/Ep ratio. It focuses on three levels of investigation:

- Seismic system comparison: Compares the Es/Ep ratio values for the same events obtained independently by two different seismic systems.

- Service provider comparison: Compares the Es/Ep values obtained from the same waveforms but using different system providers such as IMS and ESG.

- User comparison: Compares the Es/Ep values obtained from the same waveforms using the same software but processed by two different users. 


\section{METHODOLOGY}

\section{Investigation levels \\ Seismic system comparison}

To investigate the problem of consistency and transferability between different systems, two datasets were obtained from an Australian mine with two different seismic systems recording the same events. This opportunity arose when the mine needed to extend and upgrade their seismic system and, at the time, decided to change from one system provider to another. For a period of 10 months (3 May 2007 to 10 March 2008), both systems were kept operational to record the same events. After associating the events between the two databases, the consistency and transferability of the Es/Ep parameters obtained from the two systems were investigated. The seismic system comparison includes the effects of the different arrays, sensor type, sensor frequency ranges and the software and algorithms employed.

\section{Service provider comparison}

To investigate the effect of different algorithms on the Es/Ep parameters, the influence of different system and sensor configurations were eliminated. Waveform data were obtained from an open pit research site Salvoni et al (2016a) and was provided to three different organisations. Two of these organisations are commercial service providers and the other a government sponsored research organisation. The waveforms were provided to each of the organisations to process using their own algorithms and software to locate the events and calculate the source parameters. The results from the three organisations were compiled and compared.

\section{User comparison}

To investigate the user level influence on the Es/Ep parameter consistency, waveforms from the same dataset were processed using the same commercially available processing software. As the differences in the results are only due to the different user approaches to the processing and the different user skill levels, the comparison of the results provides a way of quantifying user influence.

\section{Method comparison}

For each of the investigated levels, the Es/Ep values obtained from the different datasets are displayed as scatterplots. Also added to these charts is a QQ-plot of the data. The QQ-plot simply plots different independently obtained quantile values from the datasets. The QQ-plot following a 1:1 line indicates that the two datasets have similar statistical distributions but does not imply any correlation. A change in the slope or an offset of the QQ-plot from the 1:1 line indicates a mean systematic slope difference and data offset between the two datasets. To quantify the deviation from the 1:1 line, the slope of the best fit line is calculated, as well as the difference in the mean values for the two datasets $\left(\Delta_{\text {mean }}\right)$.

In addition to these plots, the probability density functions (PDFs) of the Es/Ep values from the different databases are provided, as well as the cumulative density functions (CDFs) of the difference between the Es/Ep values from the different databases. For each of the datasets, the statistical properties are also determined (mean, standard deviation, minimum, maximum and the PDF distribution type).

In principle, if a consistent and quantifiable correlation exists between the two datasets, it would be possible to perform a general transformation from one dataset to another. This would allow an overall statistical comparison between the Es/Ep values obtained from the different systems. To evaluate the transferability between the two databases, we calculate $\mathrm{R}^{2}$ and the Pearson's correlation coefficient (CC).

The $\mathrm{R}^{2}$ value is determined from the linear best fit. Dataset 1 is taken as the independent variable whilst Dataset 2 is taken as the dependent valuable, and hence the $R^{2}$ value is calculated as follows:

$$
R^{2}=1-\frac{\sum\left(y_{D 2}-y_{m o d e l}\right)^{2}}{\sum\left(y_{D 2}-\overline{y_{D 2}}\right)^{2}}
$$


Where:

- $y_{\text {model }}$ is the best linear fit value for the source parameter

- $y_{D 2}$ is the source parameter value of Dataset 2

- $\overline{y_{D 2}}$ is the average of the source parameter values for Dataset 2.

Perfect correlation is indicated by $\mathrm{R}^{2}=100 \%$, while low values indicate poor correlation.

For the $\mathrm{CC}$, the following equation was used:

$$
C c=\frac{\operatorname{cov}(D 1, D 2)}{\sigma_{D 1} \sigma_{D 2}}
$$

Where:

- $\operatorname{cov}(D 1, D 2)$ is the covariance between Database 1 , and Database 2

- $\sigma_{D 1}$ is the standard deviation of Database 1

- $\sigma_{D 2}$ is the standard deviation of Database 2 .

Perfect positive and negative correlation is indicated with $C C=1$ and -1 respectively, with poorer correlation corresponding with smaller values. A CC $=0$ indicates that no correlation exists.

\section{RESULTS}

\section{Seismic system comparison}

Data was obtained from two different systems from two different service providers recording the same seismic events. System 1 consisted predominantly of $14 \mathrm{~Hz}$ triaxial geophones, whilst System 2 had a denser array and consisted of predominantly uniaxial accelerometers with lower frequency limits of $50 \mathrm{~Hz}$.

In order to understand the impact of this difference better, an association between the events in the two databases is required. The two systems were totally independent and did not use GPS time and, therefore, the event occurrence time differs. Time drift occurred on one or both systems and the time differences between the two systems were not constant. Also, the location of the events between the two systems differed as a result of the different arrays and location algorithms. Differences in system and sensor sensitivity also resulted in not all events being recorded in both databases. For this reason, an analysis was performed to associate events from one database to the other.

For every event in Database 1, all the events in Database 2 within a $100 \mathrm{~m}$ distance were obtained and associated with it. In this one-to-many database association, the event closest in time to the Database 1 event was assumed to be the sister event from Database 2. Figure 1a and $b$ show the spatial plots for the final associated sister events from the two systems. A distribution of the difference in time and location is shown in Figure 1c and Figure 1d. Based on this event association, the difference between the Es/Ep values obtained from the two systems can further be investigated. 

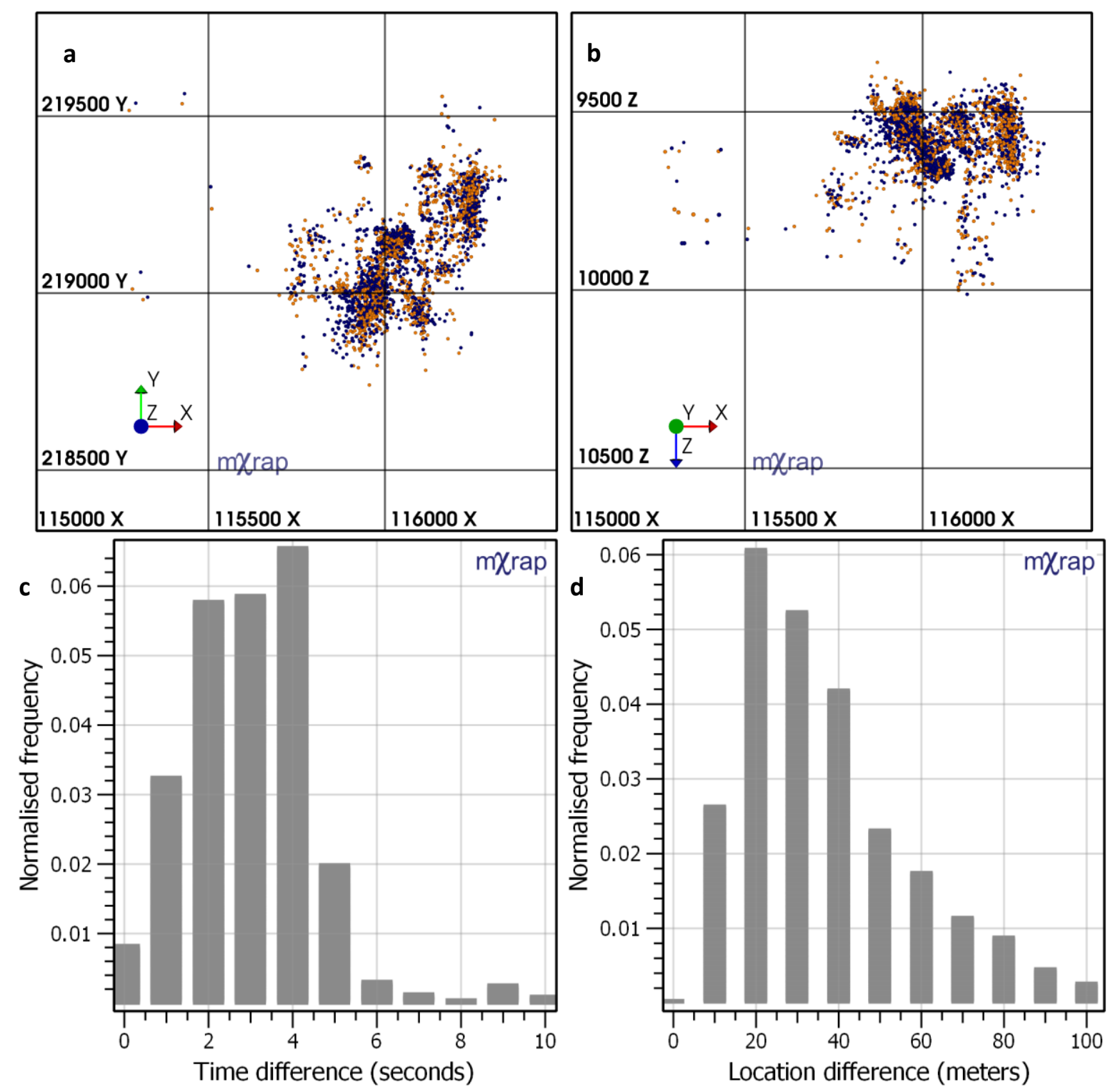

Figure 1. (a) and b) indicate the spatial plots for the two systems; System 1 is indicated by the blue dots and System 2 by the orange dots. (c) histogram of the difference in time between the associated events.

(d) the difference in time histogram

Figure 2a provides a scatter plot of Es/Ep values obtained from the two systems. From the QQ-plot and the scatter plot, it is clear that System 1 generally yielded higher values than System 2 with an offset of 0.24 ( $\Delta$ mean) also visible in the PDFs shown in Figure 2c. Apart from the offset, the PDFs for the Es/Ep values obtained from the two systems have similar shapes. However, there is a lack of correlation between the two datasets with very low $\mathrm{R}^{2}$ and $\mathrm{C}_{\mathrm{c}}$ values. Figure $2 \mathrm{~b}$ plots the CDF of the difference between Es/Ep values for the two systems indicating that more than $50 \%$ of the events have a difference of more than 10, which would result in different interpretations with current accepted practice (See Section 3.1). 


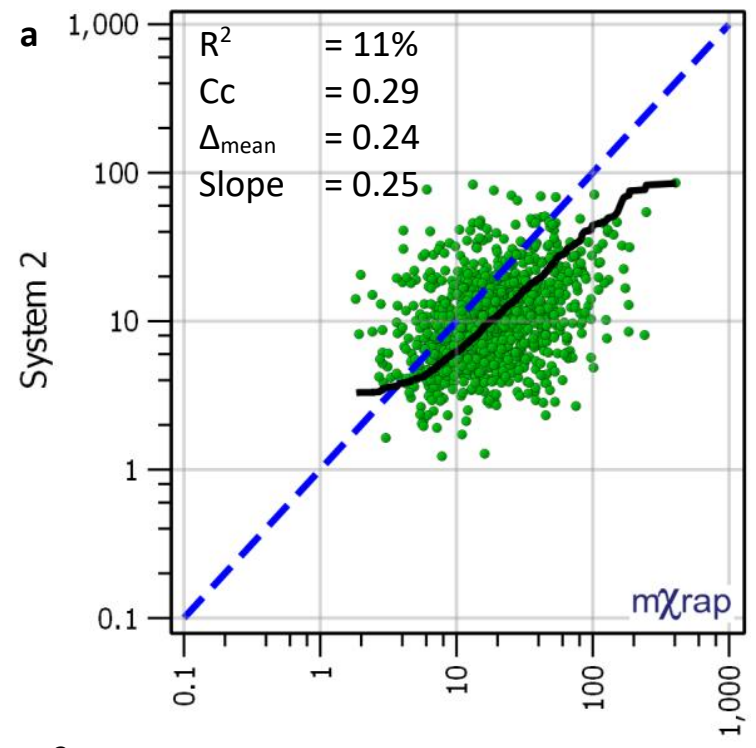

C

d

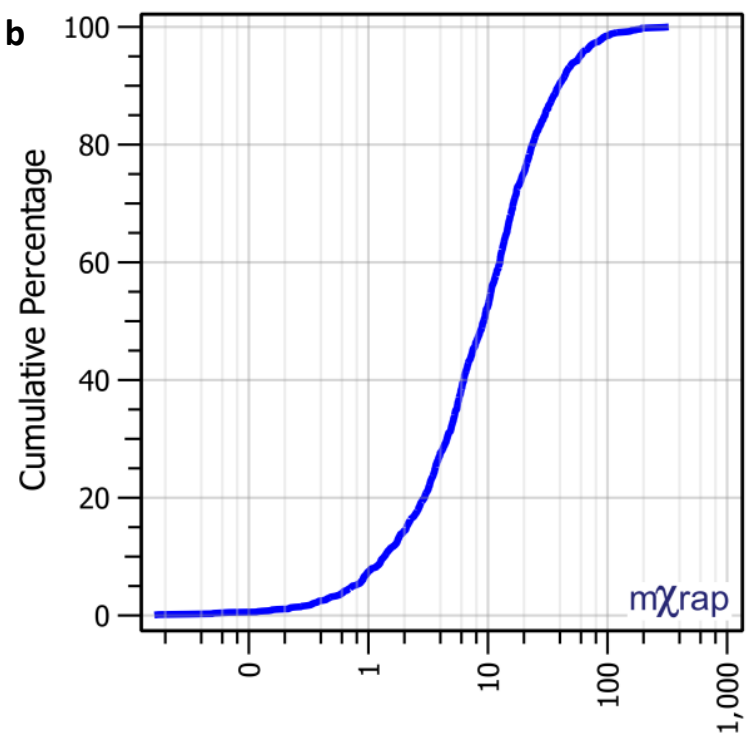

Es:Ep Difference

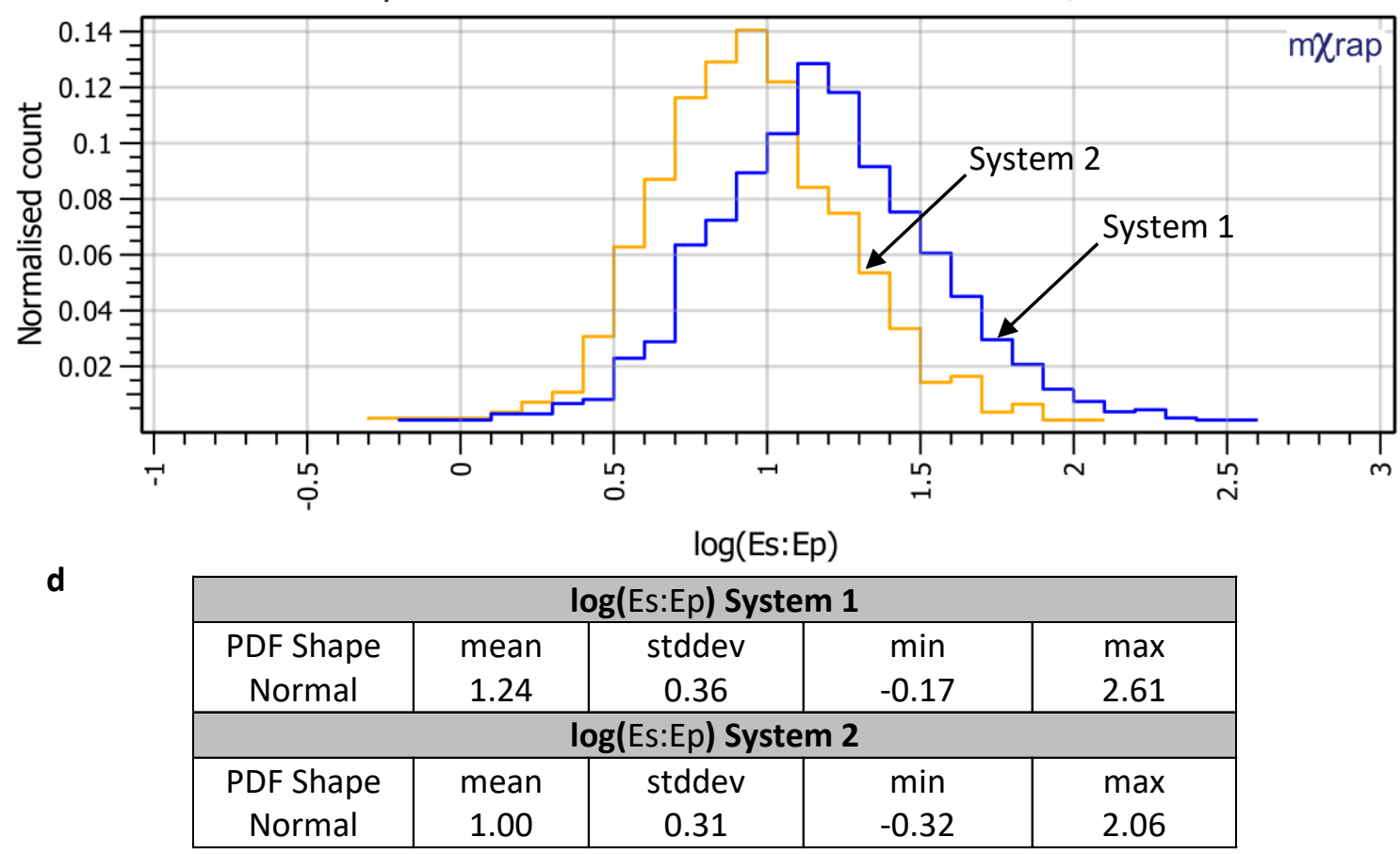

Figure 2. System 1 to System 2 comparison. (a) scatter plot of sister events. (b) CDF of the difference between the systems. (c) PDF of ES/EP from the two systems. (d) summary of the PDF properties for each system

The two systems have very different sensor distributions, and it is acknowledged that comparing a system with primarily uniaxial sensors with a system with predominantly triaxial sensors is not ideal. However, if this is the predominant reason for there being no correlation between the two systems' data, there will be an improvement in the data for the subsequent scenarios.

\section{Service provider comparison}

Waveforms recorded by a single system were processed by three service providers each using its own software/algorithms. In this case, the data was collected from an open pit mine microseismic system. More details are provided in Salvoni and Dight (2016), Salvoni et al (2016b). This study is therefore limited to a dataset consisting of smaller events and from a lower stress environment. In this section, 
the three service providers are referred to as SP1, SP2 and SP3. Figure 7 shows the total number of events accepted for the three different service providers.

The expectation here is that there should be an improvement in at least the consistency of the Es/Ep values. Any systematic error that might be introduced due to the sensor array configuration is eliminated from this comparison datasets, as the same databases of seismograms are used.

For all three service providers, velocity models were built based on one calibration blast, consisting of 7 detonations separated by approximately $10 \mathrm{~m}$. SP1 established a homogeneous and isotropic velocity model with P-wave and S-wave velocity (Vp and Vs) of $3672 \mathrm{~m} / \mathrm{s}$ and $2046 \mathrm{~m} / \mathrm{s}$ respectively. In this case, the possible effect of pit geometry on the seismic wave ray path was not considered. A standard processing method, involving triggered data based on an STA/LTA triggering algorithm was adopted. Waveforms were processed manually.

SP2 employed a two layered velocity model, where part of the pit slope had a Vp and Vs of $3700 \mathrm{~m} / \mathrm{s}$ and $1960 \mathrm{~m} / \mathrm{s}$ respectively, and the rest of the pit slope had a Vp of $2900 \mathrm{~m} / \mathrm{s}$ and Vs of $1550 \mathrm{~m} / \mathrm{s}$. The service provider in this case used the recorded continuous data and manually reprocessed all the events. The pit surface was used as a constraint to avoid events being located above the surface of the pit.

SP3 considered a homogeneous isotropic velocity model with a Vp of $3620 \mathrm{~m} / \mathrm{s}$ and a Vs of $1940 \mathrm{~m} / \mathrm{s}$. The model was further refined to consider the possible effect of pit geometry. The algorithm firstly identifies whether the theoretical straight ray path intersects the pit. If this is the case, the algorithm uses a Huygens-Fresnel principle to find the shortest path which doesn't intercept the pit (Aki and Richards, 2002). The STA/LTA ratio was lower (compared to SP1 and SP2) to ensure a larger number of sensor waveforms were considered for processing which led to a larger event database. Automatic processing of the events was adopted.

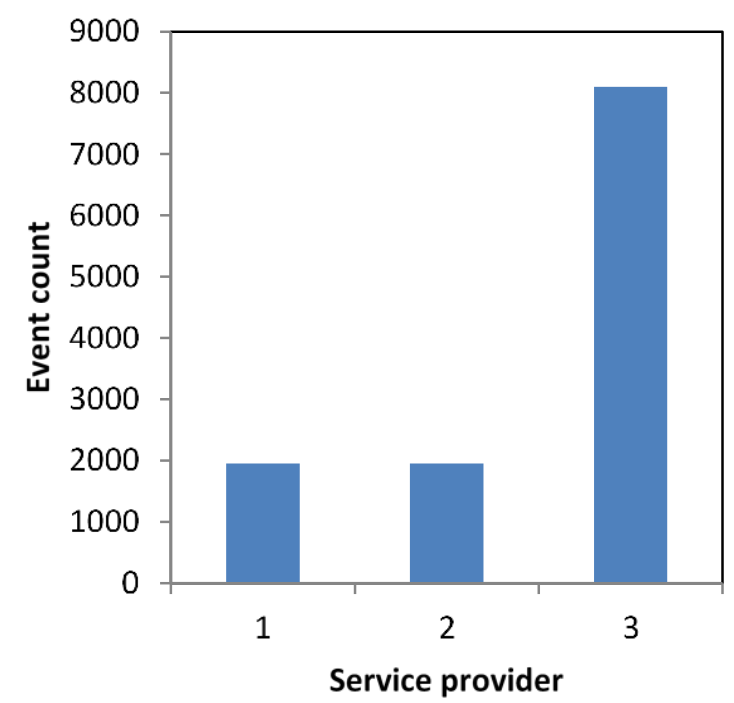

Figure 3. Event count for the three different service providers

\section{S-Wave to $P$-Wave energy ratio}

The results of the comparison of Es/Ep for SP1, SP2 and SP3 are summarised in Figure 4. The scatter plots locate around the 1:1 lines with little or no correlation as evidenced by the R2 values of $6-12 \%$. The PDFs (c) and the QQ-plots (a) following the 1:1 line indicate that the three datasets follow the same type of distribution. However, no correlation between the parameter from the databases exists. In all the cases, almost half of the data have differences greater than 3 whilst the bulk of the data varies between 1 and 10 which will result in different interpretation according to generally accepted and applied practice (See Section 3.1) 


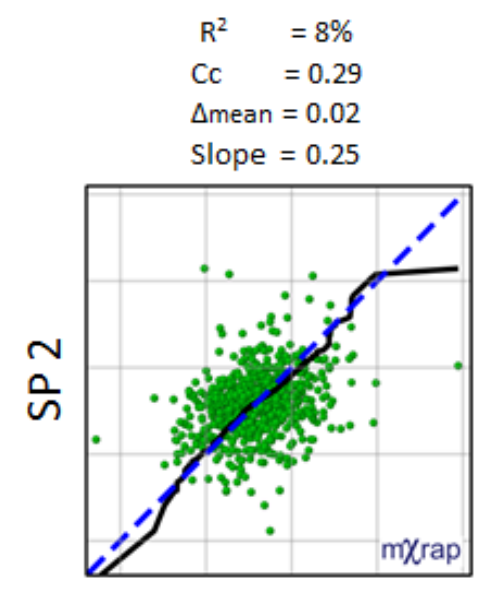

SP 1

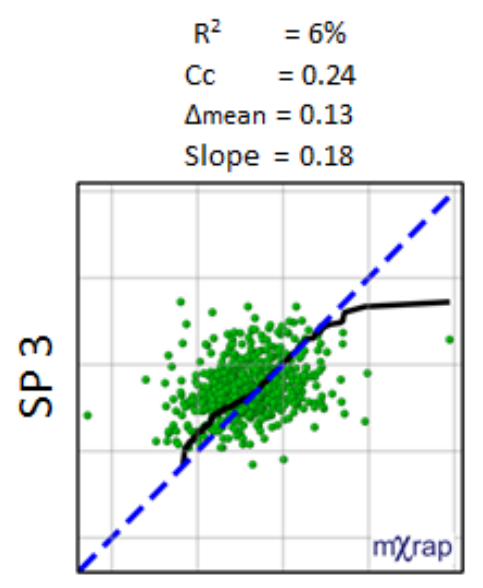

SP 1

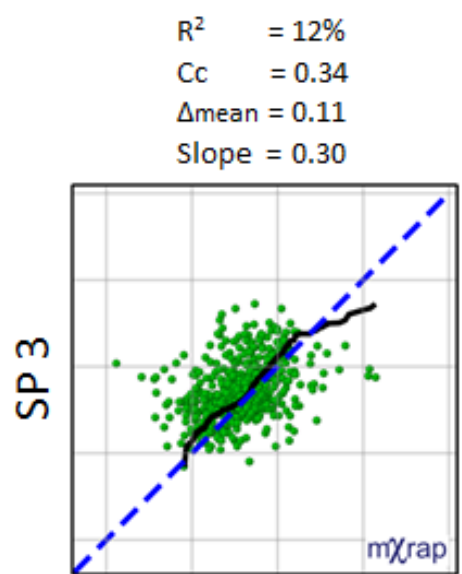

SP 2
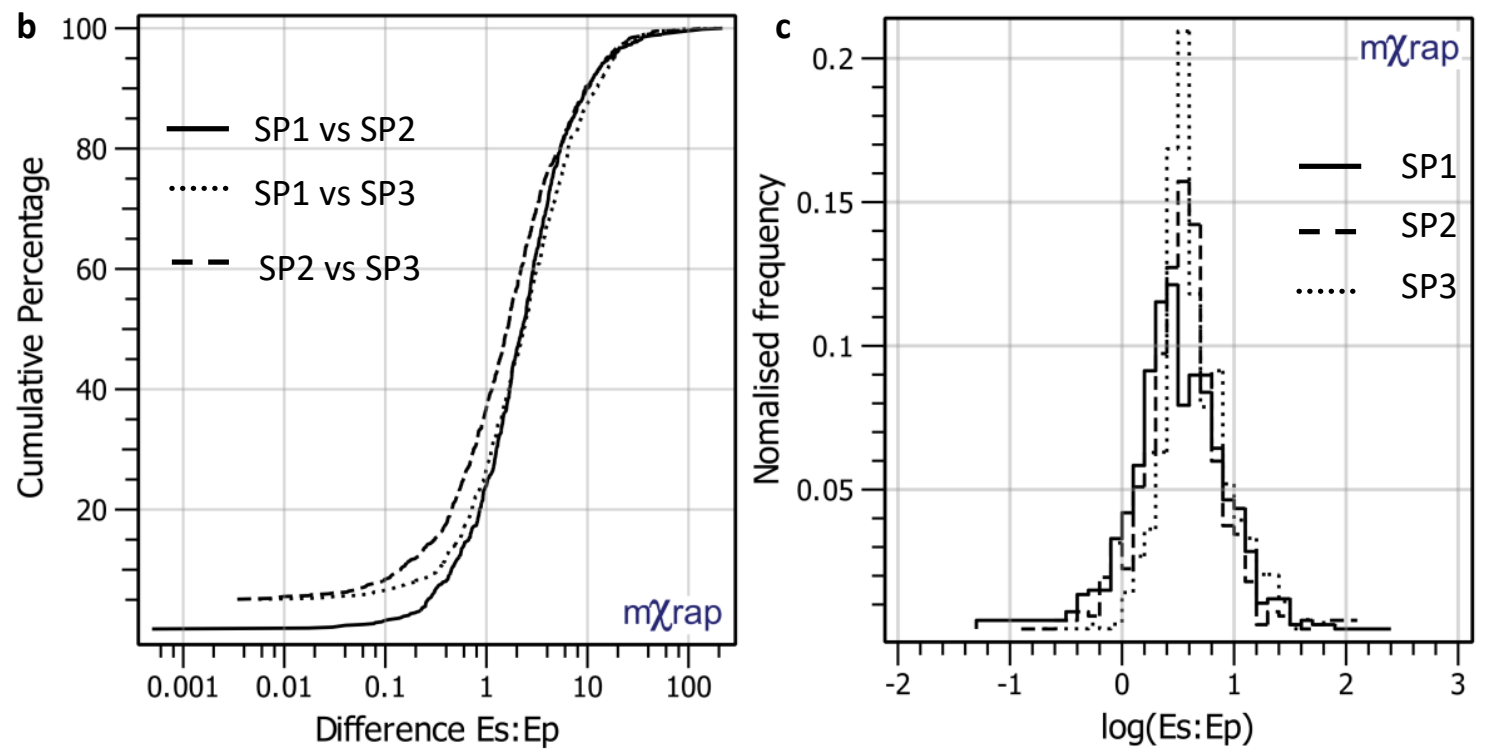

d

\begin{tabular}{|c|c|c|c|c|}
\hline \multicolumn{5}{|c|}{$\log ($ Es:Ep) System 1 } \\
\hline $\begin{array}{c}\text { PDF Shape } \\
\text { Normal }\end{array}$ & $\begin{array}{c}\text { mean } \\
0.57\end{array}$ & $\begin{array}{c}\text { stddev } \\
0.41\end{array}$ & $\begin{array}{c}\text { min } \\
-1.32\end{array}$ & max \\
\hline \multicolumn{5}{|c|}{$\log$ (Es:Ep) System 2 } \\
\hline PDF Shape & mean & stddev & $\min$ & $\max$ \\
Normal & 0.59 & 0.35 & -0.89 & 2.14 \\
\hline \multicolumn{6}{|c|}{$\log ($ Es:Ep) System 3 } \\
\hline PDF Shape & mean & stddev & min & max \\
Normal & 0.69 & 0.31 & -0.70 & 1.72 \\
\hline
\end{tabular}

Figure 4. Comparison for the three service providers. (a) scatter plots for the different service providers; the blue lines indicate the 1:1 line and the black lines the QQ-plot for the datasets. (b) CDF of the difference between the service providers. (c) PDF for each of the service providers. (d) summary of the PDF properties for each system

There is virtually no correlation existing for Es/Ep values. The differences mentioned in this section can only be attributed to the algorithms employed by the different organisations with possible added user effects. It should be noted that, although the same waveforms were provided to different organisations, 
depending on the sensors used in the processing of the events, different combinations of waveforms may be included in the calculation of the source parameters for the same event.

\section{User comparison}

Both the previous study levels included user effects. In this section, only the user effect is quantified. Waveforms from the same system are processed with the same source and location algorithm. The only difference between the two compared datasets is that the waveforms were processed by different users.

User 1 performed processing as a service done on a routine basis without any site knowledge and no personal interest in the data. User 2 is involved with the site, has an intimate knowledge of the site and has a personal interest in the data. User 2 also considers the location of seismic sensors and aims to maximise the number of sensors used.

\section{S-Wave to P-Wave energy ratio}

Figure 5 summarises the comparison between Es/Ep for the two datasets. The PDFs and the QQ-plot following the 1:1 line indicate that the two databases have very similar distributions. However, there is virtually no correlation between the two distributions as is evident from the scatter in Figure 5a and the low $\mathrm{R}^{2}$ and Cc values. More than $30 \%$ of the data has a difference of greater than five (See Figure $5 \mathrm{~b}$ ), which is high considering the ranges of the parameter. Since this difference is due solely to the different users processing the same waveforms with the same software, this result indicates the diagnostic value assigned to this parameter are not justified 

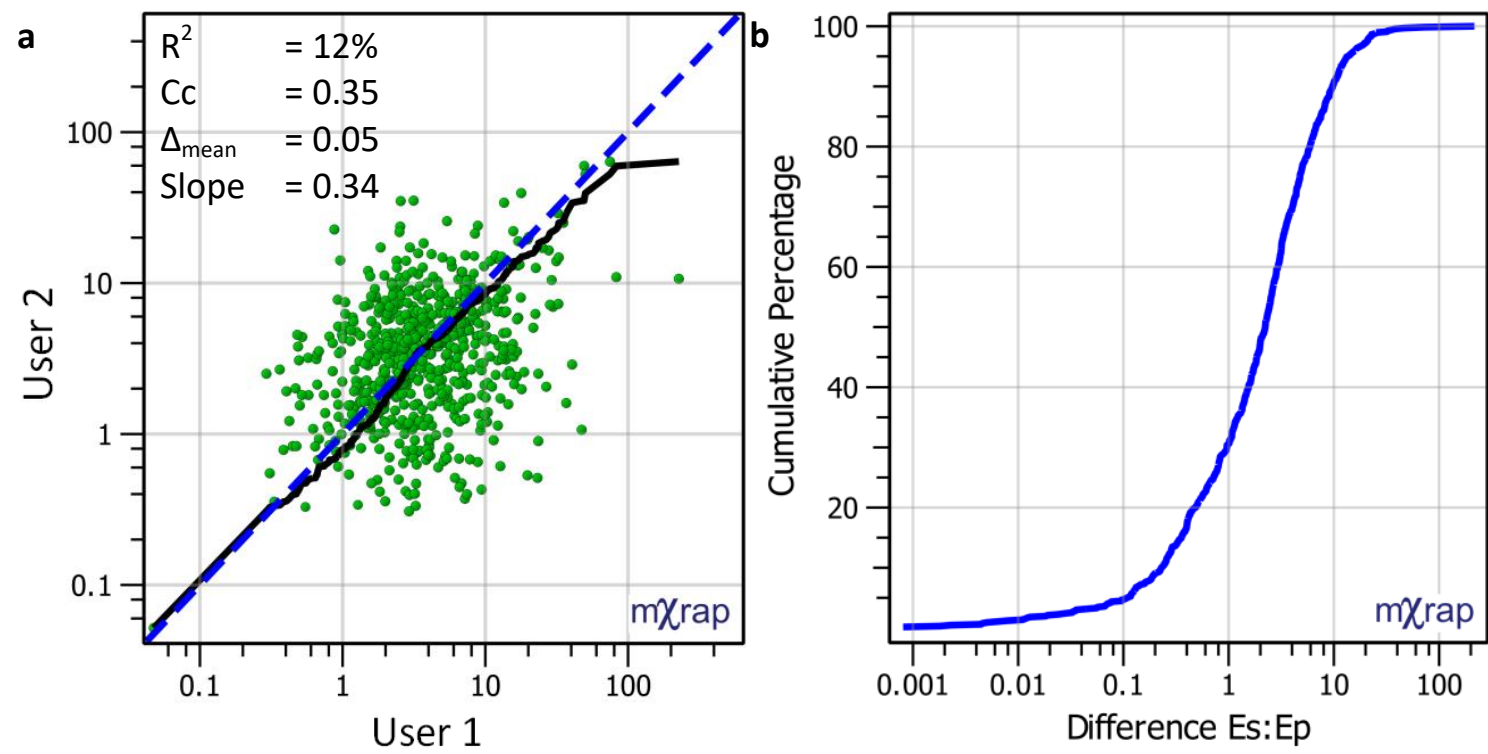

.

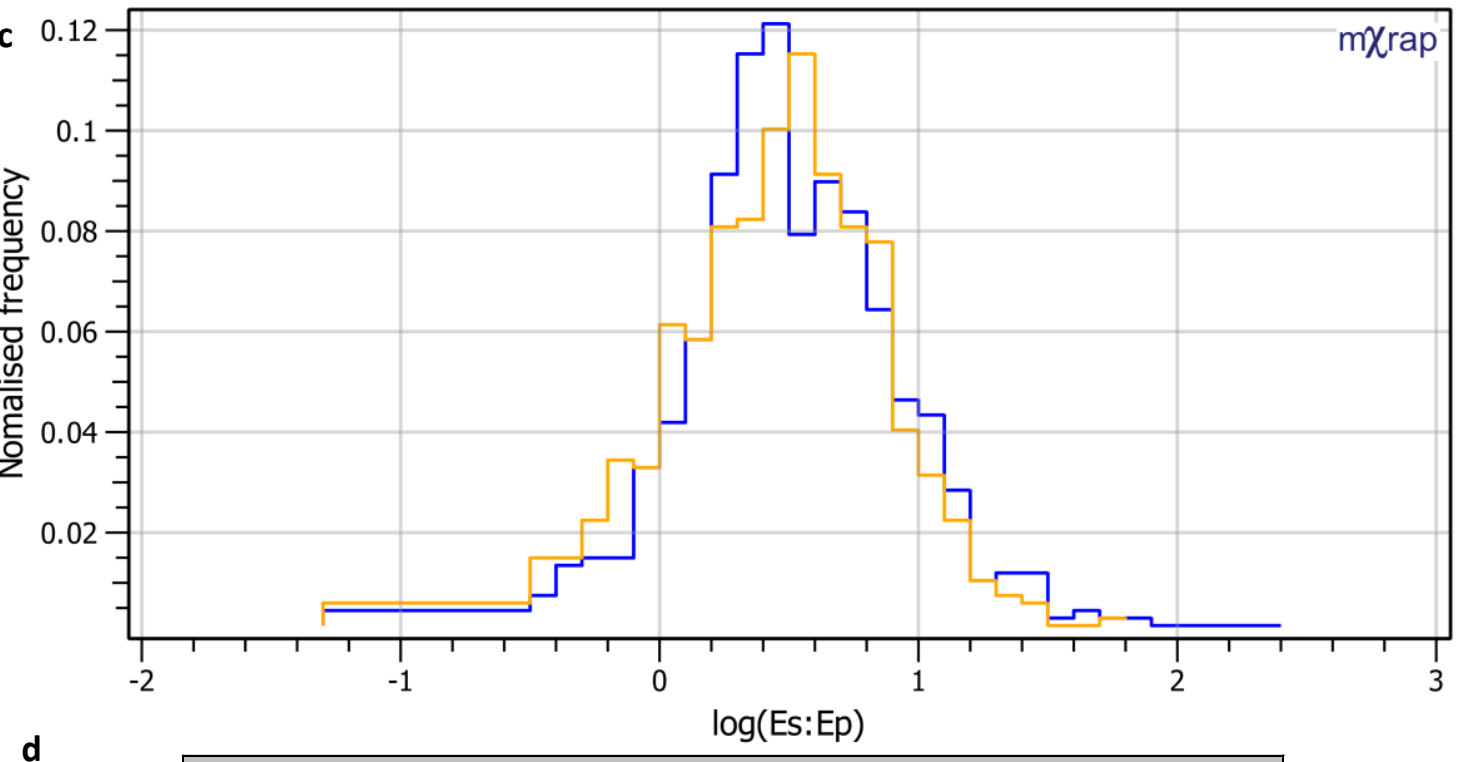

d

\begin{tabular}{|c|c|c|c|c|}
\hline \multicolumn{5}{|c|}{$\log$ (Es:Ep) User 1 } \\
\hline $\begin{array}{c}\text { PDF Shape } \\
\text { Normal }\end{array}$ & mean & stddev & $\min$ & $\max$ \\
\hline \multicolumn{5}{|c|}{$\log ($ Es:Ep) User 2} \\
\hline PDF Shape & mean & stddev & min & max \\
Beta & 0.51 & 0.41 & -1.28 & 1.81 \\
\hline
\end{tabular}

Figure 5. Comparison between the User 1 and User 2 data. (a) scatter plot for the different users; the blue dashed line indicates the one to one line and the black line the QQ plot. (b) CDF of the difference between the users (c) PDF of the two users; User 1 indicated with the blue line; User 2 indicated by the orange line. (d) summary of the PDF properties for each user

\section{DISCUSSION}

Table I summarises the results for the Es/Ep parameter for the different levels of investigation. The Es/Ep parameter is not transferable at any of the levels of investigation. Although the distribution ranges and shapes are very similar throughout, the direct correlation is weak. The reason for this is 
unclear but it indicates that the calculation of this parameter is sensitive to the introduction of systematic errors at all three study levels. As this parameter is often used as an indicator of source mechanism, a higher degree of robustness in the calculation of this parameter is required.

Table I. Summary of Es/Ep results for each of the scenarios studied

\begin{tabular}{|c|c|c|c|}
\hline Analysis result & System & Algorithm & User \\
\hline $\mathrm{R}^{2}$ & $11 \%$ & $8 \%, 6 \%, 12 \%$ & $12 \%$ \\
\hline $\mathrm{Cc}$ & 0.29 & $0.29,0.24,0.34$ & 0.35 \\
\hline Slope & 0.25 & $0.25,0.18,0.30$ & 0.34 \\
\hline$\Delta_{\text {mean }}$ & 0.24 & $0.02,0.13,0.11$ & 0.05 \\
\hline Difference at $90 \%$ & 50 & $10,10,10$ & 10 \\
\hline PDF shape & Same & Same, same, same & Different \\
\hline
\end{tabular}

The ratio of energy associated with the S-wave and P-wave is dependent on the focal mechanism at the seismic source and the ratio of S-wave energy (Es) to P-wave energy (Ep) is regarded as an important indicator of the type of focal mechanism Cai et al (1998). In pure shear, the Es is considerably larger than Ep (Es/Ep > 20). For the tensile model, Sato (1978) has shown that Ep and Es are approximately equal. Gibowicz et al (1991) and (Gibowicz and Kijko (1994) suggest that when Es/Ep < 10, the source mechanism involves a tensile failure component, and Boatwright and Fletcher (1984) suggest pure shear corresponds with Es/Ep > 10. Hudyma and Potvin (2010) suggest that for events with Es/Ep <3, the mechanism is non-shear. This is summarised in Table II.

Table II. Interpretation of Es/Ep values generally used in the mining industry

\begin{tabular}{|c|c|}
\hline Es/Ep & Mechanism interpretations \\
\hline$<1$ & Pure tensile \\
$1-3$ & Non-shear \\
$3-10$ & Mixed \\
$>10$ & Shear \\
\hline
\end{tabular}

The extent of scatter exhibited in the value comparison at all three of the different comparison levels is wide compared to the threshold values for the different mechanism classes shown in Table 2. For a large proportion of the databases, different source mechanisms would be assigned resulting from systematic differences. This is concerning as a lot of value is placed on this parameter in industry. Even more concerning is the fact that this also applies to the user comparison, which means that different mechanisms will be assigned to the same event processed by different users using the same software.

What is not clear from this study is whether the mean value of a larger population could provide a more robust indication of the general mechanism of rock mass response in a specific area in an absolute or comparative manner. In this study, the distribution of the Es/Ep for each of the sister datasets were similar. The standard deviation of the distributions may be dominated by system effects, whilst the mean value of a larger dataset could still be indicative of the dominant mechanism at the source of those events.

A cursory investigation into the literature on mining-induced seismicity reveals many studies assigning diagnostic value to Es/Ep with the second author of this paper being among these, including Sweby et al( 2006), Hudyma (2008), Wesseloo and Sweby (2008), Hudyma and Potvin (2010), Reyes-Montes et al (2010), Mendecki (2013), Disley (2014), McGaughey (2014), Rebuli and Kohler (2014), Abolfazlzadeh and Hudyma (2016), Abolfazlzadeh et al (2017), Abolfazlzadeh et al (2019). 
End users of the data are not equipped and seldom have the resources or knowledge to investigate or even question the validity of the parameters in their database. It is reasonable for the users of the data to expect a minimum level of robustness for standard and generally accepted parameters and to interpret these parameters in accordance with published studies.

The parameters in this study were all obtained in the standard ways used in the majority of the mining industry. Even though there might be differences in the approaches taken in calculating the parameters, one would expect some correlation between parameters from different approaches, yet we have found none. This fact undermines the confidence one can have in the Es/Ep parameter.

This is not criticism directed at the Es/Ep ratio at a fundamental level. Nor do we imply that the original studies suggesting the use of Es/Ep and the threshold values were flawed. We are implying that an end-user, taking the parameters at face value can now not have any confidence in the use of this parameter, unless it is restored with a study showing under what conditions and with which assessment methods it will be reliable. In other words, the end user of the data has a defeater for his believe in reliability of Es/Ep as an indicator of source mechanism.

The original and subsequent papers on the topic do not provide enough information regarding the system, software and algorithms, and as a result, we cannot restore confidence in the parameter by reverting back to the original studies.

Publication of studies in mining-induced seismicity requires more detail on the seismic systems used. This should include, for example, the frequency ranges of the sensors, some measure of planarity/spatial coverage by the array, software used for data processing and different assumptions made in the software or system configuration.

\section{CONCLUSION}

This paper investigated the inter-system seismic data consistency of mining-induced seismic data. The consistency of the data generally improved from the seismic system comparison level to the service provider comparison level to the user comparison level as each higher level includes the systematic error from lower levels.

Inconsistencies between the datasets may have a large impact on the interpretation and correctness of data analysis methods used in any quantitative assessment of mining-induced seismicity. Such techniques might have been successfully employed on a specific dataset within the context of sitespecific experience and calibration, provided that the system does not change. Such knowledge would, however, not be transferable to another dataset.

This is especially true for the Es/Ep parameters, which seems to be highly inconsistent, where significant changes occur even at the user level study. These changes for all source parameters investigated are large enough to completely alter interpretation on an event-to-event basis. The Es/Ep is often used in the mining industry as an indicator of the source mechanism. This parameter, used on a single event, appears to be unreliable and should not be used. This study cannot make definitive conclusions on the diagnostic value of the mean Es/Ep value for larger datasets used on a comparative basis. This study, however, undermines the confidence in the diagnostic value of Es/Ep within the mining industry. The confidence in this parameter can only be restored after improving and demonstrating its robustness.

A sad consequence of this study is that it undermines the confidence in many previously performed studies simply because it is not possible to evaluate the correctness/robustness of the Es/Ep values used in these studies. Publication of studies in mining-induced seismicity requires more detail on the seismic systems used. This should include, for example, the frequency ranges of the sensors, some measure of planarity/spatial coverage by the array, software used for data processing and different assumptions made in the software or system configuration. 


\section{ACKNOWLEDGEMENTS}

We acknowledge the support of the mXrap Consortium and the Australian Centre for Geomechanics during this project.

\section{REFERENCES}

Abolfazlzadeh, Y. and Hudyma, M. (2016). Identifying and Describing a Seismogenic Zone in a Sublevel Caving Mine. Rock Mechanics and Rock Engineering, 1-17.

Abolfazlzadeh, Y., Penhall, S.L. and McKinnon, S.D. (2017). Statistical Analysis of the Outlier Events from Seismic Stress Inversion. Proceedings of Proceedings of the First International Conference on Underground Mining Technology. Hudyma, M. and Potvin, Y., (eds.), Perth, Australian Centre for Geomechanics, pp. 269-277.

Abolfazlzadeh, Y., Smith-Bougher, L., Anderson, Z., Jalbout, A. and Mataseje, A. (2019). Calibration of a Seismic Hazard Assessment Tool Using Velocity Fields and Geotechnical Data. Proceedings of Proceedings of the First International Conference on Mining Geomechanical Risk. Wesseloo, J., (ed.), Perth, Australian Centre for Geomechanics, pp. 233-244.

Aki, K. and Richards, P.G. (2002). Quantitative Seismology.

Boatwright, J. and Fletcher, J.B. (1984). The Partition of Radiated Energy between P and S Waves. Bulletin of the Seismological Society of America, 74 (2), 361-376.

Cai, M., Kaiser, P.K. and Martin, C.D. (1998). A Tensile Model for the Interpretation of Microseismic Events near Underground Openings. Seismicity Caused by Mines, Fluid Injections, Reservoirs, and Oil Extraction. Springer.

Disley, N.V. (2014). Seismic Risk and Hazard Management at Kidd Mine. Proceedings of Proceedings of the Seventh International Conference on Deep and High Stress Mining. Hudyma, M. and Potvin, Y., (eds.), Perth, Australian Centre for Geomechanics, pp. 107-121.

Gibowicz, S., Young, R., Talebi, S. and Rawlence, D. (1991). Source Parameters of Seismic Events at the Underground Research Laboratory in Manitoba, Canada: Scaling Relations for Events with Moment Magnitude Smaller Than- 2. Bulletin of the Seismological Society of America, 81 (4), 11571182.

Gibowicz, S.J. and Kijko, A. (1994). An Introduction to Mining Seismology, First, Academic Press, Inc.

Hudyma, M. and Potvin, Y.H. (2010). An Engineering Approach to Seismic Risk Management in Hardrock Mines. Rock Mechanics and Rock Engineering, 43 (6), 891-906.

Hudyma, M.R. (2008). Analysis and Interpretation of Clusters of Seismic Events in Mines. PhD, University of Western Australia.

McGaughey, J., McLeod, R. and Pears, G. (2007). Integrated, Real-Time, 3d Gis-Based Geotechnical Hazard Assessment. Proceedings of 1st Canada-US Rock Mechanics Symposium, Rock Mechanics Meeting Society's Challenges and Demands. Eberhardt, E., Stead, D. and Tom, M., (eds.), London, Taylor \& Francis Group, pp. 21-28.

McGaughey, W.J. (2014). 4d Data Management and Modelling in the Assessment of Deep Underground Mining Hazard. Proceedings of Proceedings of the Seventh International Conference on Deep and High Stress Mining. Hudyma, M. and Potvin, Y., (eds.), Perth, Australian Centre for Geomechanics, pp. 93-106. 
Mendecki, A.J. (2013). Mine Seismology: Glossary of Selected Terms. Proceedings of Eighth International Symposium on Rockbursts and Seismicity in Mines, RaSiM8. Malovichko, A. and Malovichko, D., (eds.), Geophysical Survey of Russian Academy of Sciences, Mining Institute of Ural Branch of Russian Academy of Sciences, pp. 527-551.

Nordström, E., Dineva, S. and Nordlund, E. (2017). Source Parameters of Seismic Events Potentially Associated with Damage in Block 33/34 of the Kiirunavaara Mine (Sweden). Acta Geophysica.

Rebuli, D.B. and Kohler, S.J. (2014). Using Clustering Algorithms to Assist Short-Term Seismic Hazard Analysis in Deep South African Mines. Proceedings of Proceedings of the Seventh International Conference on Deep and High Stress Mining. Hudyma, M. and Potvin, Y., (eds.), Perth, Australian Centre for Geomechanics, pp. 699-708.

Reyes-Montes, J.M., Sainsbury, B.L., Pettitt, W.S., Pierce, M. and Young, R.P. (2010). Microseismic Tools for the Analysis of the Interaction between Open Pit and Underground Developments. Proceedings of Proceedings of the Second International Symposium on Block and Sublevel Caving. Potvin, Y., (ed.), Perth, Australian Centre for Geomechanics, pp. 119-131.

Salvoni, M. and Dight, P.M. (2016). Rock Damage Assessment in a Large Unstable Slope from Microseismic Monitoring-Mmg Century Mine (Queensland, Australia) Case Study. Engineering Geology, Vol. 210, pp. 45-56.

Salvoni, M., Morkel, I.G. and Dight, P.M. (2016a). Microseismic Data - a Comparison between Routine Trigger Method and Continuous Data Processing. Proceedings of Proceedings of 1st Asia Pacific Slope Stability in Mining Conference. Dight, P.M., (ed.).

Salvoni, M., Morkel, I.G. and Dight, P.M. (2016b). Microseismic Data - a Comparison between Routine Trigger Method and Continuous Data Processing. Proceedings of $1^{\text {st }}$ Asia Pacific Slope Stability in Mining Conference in Brisbane, Australia, 6-8 September 2016, pp. 639-656.

Sato, T. (1978). A Note on Body Wave Radiation from Expanding Tension Crack.

Sweby, G., Trifu, C., Goodchild, D. and Morris, L. (2006). High Resolution Seismic Monitoring at Mt Keith Open Pit Mine. Proceedings of Golden Rocks 2006, The 41st U.S. Symposium on Rock Mechanics (USRMS). American Rock Mechanics Association, pp. 6.

Wesseloo, J. and Sweby, G.J. (2008). Microseismic Monitoring of Hard Rock Mine Slopes. Proceedings of First Southern Hemisphere International Rock Mechanics Symposium, SHIRMS 2008. Potvin, Y., Carter, J., Dyskin, A. and Jeffrey, R., (eds.), Perth, Western Australia, Australian Centre for Geomechanics, pp. 433-450. 


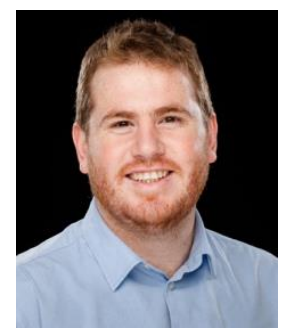

\section{Gerhard Morkel}

Research Engineer

Australian Centre for Geomechanics

Gerhard graduated from the North-West University, South Africa with an MSc in Physics in 2008. After finishing his studies, Gerhard joined IMS, working on the operational aspects of mine seismology. In 2009, he joined Anglogold Ashanti, where he was involved with the seismological aspects of their Western Deep level mines. During this time, Gerhard completed his Chamber of Mines (South Africa) certificates in Strata Control and Rock Mechanics (metalliferous). In 2012, Gerhard relocated to Australia where he worked as a mining seismologist, geotechnical engineer for Barrick's Kanowna Belle Mine. Gerhard joined the ACG in March 2014 as a research engineer for the ACG's mXrap project Gerhard has significant experience in the seismological and geotechnical aspects of seismically active underground mines. 